\title{
Energy-maximising control of wave energy converters using a moment-domain representation
}

\author{
Nicolás Faedo ${ }^{\mathrm{a}, *}$, Giordano Scarciotti ${ }^{\mathrm{b}}$, Alessandro Astolfi ${ }^{\mathrm{b}, \mathrm{c}}$, John V. Ringwood ${ }^{\mathrm{a}}$ \\ a Centre for Ocean Energy Research, Maynooth University, Maynooth, Co.Kildare, Ireland \\ b Department of Electrical and Electronic Engineering, Imperial College London, London SW7 2AZ, UK \\ ' Department of Civil Engineering and Computer Science Engineering, University of Rome "Tor Vergata", 00133 Rome, Italy
}

\section{A R T I C L E I N F O}

\section{Keywords:}

Optimal control

Wave energy converter

Phasor transform

Energy maximisation

Moment domain

\begin{abstract}
A B S T R A C T
Wave Energy Converters (WECs) have to be controlled to ensure maximum energy extraction from waves while considering, at the same time, physical constraints on the motion of the real device and actuator characteristics. Since the control objective for WECs deviates significantly from the traditional reference "tracking" problem in classical control, the specification of an optimal control law, that optimises energy absorption under different sea-states, is non-trivial. Different approaches based on optimal control methodologies have been proposed for this energy-maximising objective, with considerable diversity on the optimisation formulation. Recently, a novel mathematical tool to compute the steady-state response of a system has been proposed: the moment-based phasor transform. This mathematical framework is inspired by the theory of model reduction by moment-matching and considers both continuous and discontinuous inputs, depicting an efficient and closed-form method to compute such a steady-state behaviour. This study approaches the design of an energy-maximising optimal controller for a single WEC device by employing the moment-based phasor transform, describing a pioneering application of this novel moment-matching mathematical scheme to an optimal control problem. Under this framework, the energy-maximising optimal control formulation is shown to be a strictly concave quadratic program, allowing the application of well-known efficient real-time algorithms.
\end{abstract}

\section{Introduction}

Energy capture from ocean waves has the potential to help fulfil the increasing worldwide energy demand, with an estimation of about 32.000 TWh/year (Mork, Barstow, Kabuth, \& Pontes, 2010). Despite such a potential, wave energy is still at an early stage of development, since the technical and conceptual convergence to a technology best suited for this application has not yet been achieved (Edenhofer et al., 2011). Consequently, hundreds of patents, proposing different methodologies, have been filled all over the world (Pelc \& Fujita, 2002). A noteworthy overview and classification of Wave Energy Converters (WECs) can be found in Falcão (2010).

In a more precise definition, a WEC is a device to harvest ocean wave energy by converting the mechanical energy of the waves to electrical energy by means of a Power Take-Off (PTO) system. In order to be profitable, an optimised process that ensures extracting the maximum time averaged power, for a given WEC device, from ocean waves is crucial. Moreover, in order to maximise power absorption and minimise the risk of damage, such an optimisation strategy must take into account the physical limitations of the whole conversion chain. Such an optimisation procedure can be achieved by designing an optimal controller that accomplishes such objectives.

A considerable number of optimal control formulations and methods have been studied and developed to maximise the energy extraction process from WECs, with extensive reviews available, for example in Ringwood, Bacelli, and Fusco (2014). One particular popular wave energy control strategy is Model Predictive Control (MPC). The success of MPC on the energy-maximising control is mainly due to its ability to handle physical constraints systematically and within a finite horizon optimisation process. While MPC applied to WECs also involves a mathematical model, a typical receding horizon strategy, and can deal with system constraints, the objective function contrasts significantly with the one related to the usual set-point tracking objective. Rather, a converted energy-maximising objective, consistent with the definition

\footnotetext{
* Corresponding author.

E-mail address: nicolas.faedo.2017@mumail.ie (N. Faedo).
} 
of the WEC control problem (see Section 3.3) is employed. In particular, this variation can cause numerical search problems, due to a potential loss of convexity of the performance function involved for this application (Faedo, Olaya, \& Ringwood, 2017), compared to the normal quadratic form associated with tracking problems. In addition, the computational burden required for such a strategy can render the controller unsuitable for real-time applications (Faedo et al., 2017). Motivated by the appealing characteristics of MPC, several studies utilise "MPC-like" strategies, based on spectral and pseudospectral methods (Fahroo \& Ross, 2008; Garg, Hager, \& Rao, 2011), to try to overcome the (possibly) demanding computational effort of the original MPC optimal control formulation. A recent overview of both MPC and spectral and pseudospectral MPC-like strategies in wave energy applications can be found in Faedo et al. (2017). Notwithstanding, computing this energy-maximising control law in real-time is currently a strong concern among the wave energy community, and most of the proposed real-time strategies are usually inherently suboptimal.

Since the sea state (which directly affect the dynamic behaviour of WECs) varies slowly over time, the steady-state analysis of WECs is of paramount importance to design efficient real-time controllers for energy maximisation, as already exploited in studies such as Bacelli and Ringwood (2015) or Bacelli, Ringwood, and Gilloteaux (2011). Recently the moment-based phasor transform has been proposed to compute the steady-state response of a dynamical system under continuous or discontinuous inputs, see Scarciotti and Astolfi (2016b). From now on we refer to the framework induced by the moment-based phasor transform as the moment-domain characterisation (or formulation) of a system. This mathematical tool is based upon the theory developed in several studies concerning model order reduction (and particularly, momentmatching methods), such as Astolfi (2010) and Scarciotti and Astolfi (2015, 2016a).

In particular, in Scarciotti and Astolfi (2016b) it has been shown that the phasors of an electrical circuit are the moments computed at a single point on the imaginary axis of the transfer function of the linear system describing the circuit. Exploiting this relation, Scarciotti and Astolfi (2016b) has developed a mathematical framework to perform the steady-state analysis of systems driven by both continuous and discontinuous sources. The use of this framework is demonstrated in Scarciotti and Astolfi (2016b), both analytically and numerically, by analysing the steady-state behaviour of power inverters and wireless power transfer systems.

Nevertheless, and to the best of the authors knowledge, this momentbased framework has not yet been exploited to solve an optimal control problem. In this paper, we recognise the potential of such a mathematical tool to present a first application of the moment-based phasor transform for optimal control design, subject to path constraints. In particular, an energy-maximising optimal controller for a wave energy converter is designed, based on such a novel framework. Moreover, since the theoretical formulation is presented for a general class of devices, this paper not only demonstrates a single application case, but introduces the mathematical foundations for a novel approach to modelbased optimal control design for WECs, in general.

The remainder of this study is organised as follows: first, basics of the moment representation of a system and its connection with the steady-state behaviour of a dynamical system are recalled in Section 2, while the WEC optimal control problem is described in Section 3. A novel moment-based approach for the solution of the optimal control problem for WECs is developed analytically in Section 4, constituting the main original contribution of the paper. Numerical examples of the application of the moment-based WEC control formulation, under different sea conditions, are given in Section 5, proving the efficacy of the approach, while conclusions on the overall application of the proposed method are provided in Section 6.

\subsection{Notation and preliminaries}

Standard notation is considered through this study, with some exceptions further detailed in this preliminary section. $\mathbb{R}^{+}\left(\mathbb{R}^{-}\right)$denotes the set of non-negative (non-positive) real numbers. $\mathbb{C}^{0}$ denotes the set of pure-imaginary complex numbers and $\mathbb{C}^{-}$denotes the set of complex numbers with negative real part. The symbol 0 stands for any zero element, according to the context. The symbol $\mathbb{I}_{n}$ denotes an order $n$ identity matrix, while the notation $\mathbb{1}_{n \times m}$ is used to denote a $n \times m$ Hadamard identity matrix (i.e. a $n \times m$ matrix with all its entries equal to 1). The spectrum of a matrix $A \in \mathbb{R}^{n \times n}$, i.e. the set of its eigenvalues, is denoted as $\sigma(A)$. The symbol $\bigoplus$ denotes the direct sum of $n$ matrices, i.e. $\bigoplus_{i=1}^{n} A_{i}=\operatorname{diag}\left(A_{1}, A_{2}, \ldots, A_{n}\right)$. The notation $\mathfrak{R}\{z\}$ and $\mathfrak{I}\{z\}$, with $z \in \mathbb{C}$, stands for the real-part and the imaginary-part operators respectively, whilst $\mathscr{H}\{Z\}=\frac{Z+Z \mathrm{~T}}{2}$ stands for the symmetricpart of $Z$, where $Z \in \mathbb{R}^{n \times n}$. If $F \in \mathbb{R}^{n \times n}$ is a symmetric matrix, the expression $F>0$ implies that $F$ is positive-definite. The Kronecker product between two matrices $M_{1} \in \mathbb{R}^{n \times m}$ and $M_{2} \in \mathbb{R}^{p \times q}$ is denoted as $M_{1} \otimes M_{2} \in \mathbb{R}^{n p \times m q}$. The convolution between two functions $f(t)$ and $g(t)$ over a finite range $[0, t]$, i.e. $\int_{0}^{t} f(\tau) g(t-\tau) d \tau$ is denoted as $f * g$. The inner product between two functions $w(t), l(t) \in L^{2}(\mathbb{R})[a, b]$, where $L^{2}(\mathbb{R})[a, b]$ is the set of all real-valued functions square integrable in the interval $[a, b]$, is given by

$\langle w(t), l(t)\rangle=\int_{a}^{b} w(\tau) l(\tau) d \tau$

If $\Omega: \mathscr{X} \longrightarrow \mathscr{Z}$ is a linear transformation, where $\mathscr{X}$ and $\mathscr{Z}$ are $\mathbb{K}$-vector spaces ( $\mathbb{K}$ a field), the image and the kernel of $\Omega$ are denoted $\operatorname{Im}\{\Omega\} \subset \mathscr{Z}$ and $\operatorname{Ker}\{\Omega\} \subset \mathscr{X}$, respectively. Finally, the symbol $\varepsilon_{n} \in \mathbb{R}^{n \times 1}$ denotes a vector with odd components equal to 1 and even components equal to 0 .

In the remainder of this section the formal definitions of two important operators are presented, since their definition in the literature can be often ambiguous.

Definition 1 (Brewer, 1978 Kronecker Sum). The Kronecker sum between two matrices $P_{1}$ and $P_{2}$, with $P_{1} \in \mathbb{R}^{n \times n}$ and $P_{2} \in \mathbb{R}^{k \times k}$, is defined (and denoted) as

$P_{1} \hat{\oplus} P_{2} \triangleq P_{1} \otimes \mathbb{I}_{k}+\mathbb{I}_{n} \otimes P_{2}$.

Definition 2 (Brewer, 1978 Vec Operator). Given a matrix $H=$ $\left[h_{1}, h_{2}, \ldots, h_{n}\right] \in \mathbb{R}^{n \times m}$, where $h_{j} \in \mathbb{R}^{n}, j=1, \ldots, m$, the vector valued operator vec is defined as

$\operatorname{vec}\{H\} \triangleq\left[\begin{array}{c}h_{1} \\ h_{2} \\ \vdots \\ h_{m}\end{array}\right] \in \mathbb{R}^{n m}$.

Finally, useful theorems and properties of the Kronecker sum, and the vec and Hermitian-part operators, are recalled in the following.

Theorem 1 (Brewer, 1978). Consider matrices $P_{1}$ and $P_{2}$ as in Definition 1. Assume that $P_{1}$ and $P_{2}$ have eigenvalues $\lambda_{i}$, for $i=1, \ldots, n$, and $\mu_{j}$, for $j=1, \ldots, k$. Then the Kronecker sum $P_{1} \hat{\oplus} P_{2}$ has the nk eigenvalues

$\lambda_{1}+\mu_{1}, \ldots, \lambda_{1}+\mu_{k}, \lambda_{2}+\mu_{1}, \ldots, \lambda_{2}+\mu_{k}, \ldots, \lambda_{n}+\mu_{k}$.

Corollary 1 (Brewer, 1978). The Kronecker sum $P_{1} \hat{\oplus} P_{2}$ is invertible if and only if $\sigma\left(P_{1}\right) \cap \sigma\left(-P_{2}\right)=\emptyset$.

Property 1 (Brewer, 1978). Let $A \in \mathbb{R}^{n \times m}$ and $B \in \mathbb{R}^{p \times q}$. The following relation for the vec operator holds:

$\operatorname{vec}\{A B\}=\left(\mathbb{I}_{q} \otimes A\right) \operatorname{vec}\{B\}=\left(B^{\top} \otimes \mathbb{I}_{n}\right) \operatorname{vec}\{A\}$. 
Property 2 (Zhang, 2011). Consider a general quadratic form given by $g^{\top} M g \in \mathbb{R}$, where $g \in \mathbb{R}^{n \times 1}$ and $M \in \mathbb{R}^{n \times n}$. The equality

$g^{\top} M g=\frac{1}{2} g^{\top} \mathscr{H}\{M\} g$

holds.

\section{Moment-based description of a system}

The moment-based formulation, as considered in this study, was first formulated in Astolfi (2010), with the purpose of developing reducedorder models for linear and non-linear dynamical systems. Subsequent studies, such as, for example, Scarciotti and Astolfi (2015, 2016a), exploit this moment characterisation to obtain new results regarding the model reduction problem, under diverse assumptions. Furthermore, this mathematical foundation is extrapolated to the analysis of the steady-state behaviour of dynamical systems in Scarciotti and Astolfi (2016b), with the development of the moment-based phasor transform. Particularly, Scarciotti and Astolfi (2016b) illustrates the potential of this new mathematical tool by analysing power electronic devices, such as power inverters and wireless power transfer systems. A brief summary of the key elements of this moment-based theory is presented in the following.

\subsection{Moments for linear systems}

In this subsection the notion of moment for linear systems, as formulated in Astolfi (2010), is recalled. Consider a finite-dimensional, single-input, single-output, continuous-time system described, for $t \geq 0$, by the state-space model

$$
\begin{aligned}
\dot{x}(t) & =A x(t)+B u(t), \\
y(t) & =C x(t),
\end{aligned}
$$

where $x(t) \in \mathbb{R}^{n \times 1}, u(t) \in \mathbb{R}, y(t) \in \mathbb{R}, A \in \mathbb{R}^{n \times n}, B \in \mathbb{R}^{n \times 1}$ and $C \in \mathbb{R}^{1 \times n}$. Consider the associated transfer function

$H(s)=C\left(s \mathbb{I}_{n}-A\right)^{-1} B$

and assume that (7) is controllable and observable.

Definition 3 (Antoulas, 2005). The 0-moment of system (7) at $s_{i} \in \mathbb{C}$ is the complex number $\eta_{0}\left(s_{i}\right)=C\left(s_{i} \mathbb{I}_{n}-A\right)^{-1} B$. The $k$-moment of system (7) at $s_{i} \in \mathbb{C}$ is the complex number

$\eta_{k}\left(s_{i}\right)=\frac{(-1)^{k}}{k !}\left[\frac{d^{k}}{d s^{k}}\left(C\left(s \mathbb{I}_{n}-A\right)^{-1} B\right)\right]_{s=s_{i}}$,

with $k \geq 1$ integer.

In Astolfi (2010) it is shown that the moments of system (7) are in a one-to-one relation with the steady-state response (provided it exists) of the output of the interconnection between a signal generator and system (7). This result is recalled in the following theorem.

Theorem 2 (Astolfi, 2010; Scarciotti \& Astolfi, 2017). Consider system (7) and the signal generator

$$
\begin{gathered}
\dot{\xi}(t)=S \xi(t), \\
u(t)=L \xi(t),
\end{gathered}
$$

with $\xi(t) \in \mathbb{R}^{\nu \times 1}, S \in \mathbb{R}^{\nu \times v}, L \in \mathbb{R}^{1 \times v}$ and $\xi(0) \in \mathbb{R}^{\nu \times 1}$. Assume that the triple $(S, \xi(0), L)$ is minimal, $\sigma(A) \subset \mathbb{C}^{-}, \sigma(S) \subset \mathbb{C}^{0}$ and the eigenvalues of $S$ are simple. Let $\Pi \in \mathbb{R}^{n \times v}$ be the (unique) solution of the Sylvester equation

$A \Pi+B L=\Pi S$.

Then there exists a one-to-one relation between the moments $\eta_{0}\left(s_{1}\right), \eta_{0}\left(s_{2}\right)$, $\ldots, \eta_{0}\left(s_{v}\right)$, with $s_{i} \in \sigma(S)$ for all $i=1, \ldots, v$, and the steady-state response

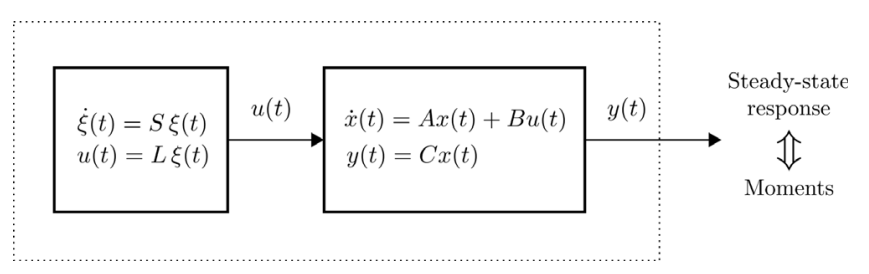

Fig. 1. Schematic of the interconnection between the system (7) and the signal generator (10).

Source: Adapted from Astolfi (2010).

$C \Pi \xi$ of the output $y$ of the interconnection of system (7) with the signal generator (10) (as in Fig. 1). In fact, the moments are uniquely determined by the matrix $C \Pi$.

Note that the minimality of the triple $(S, \xi(0), L)$ implies the observability of the pair $(S, L)$ and the excitability of the pair $(S, \xi(0))$ (see Padoan, Scarciotti, \& Astolfi, 2017 and Scarciotti \& Astolfi, 2017 for further detail). Finally, in Scarciotti and Astolfi (2016b), using the results provided by Theorem 2, a direct equivalence between phasor analysis and the moment characterisation of a system is proposed, conceiving a novel way to analyse and compute the steady-state response of a system under both continuous and discontinuous excitation. In particular, Scarciotti and Astolfi (2016b) has shown that the classical phasor transform (Davis, 1998; Nilsson \& Riedel, 2015) of a circuit can be associated to a Sylvester equation. A few definitions and fundamental properties regarding this mathematical framework are recalled in the subsequent paragraph. For the sake of clarity, the precise definition of the phasor transform of a linear system is presented in the following.

Definition 4 (Scarciotti \& Astolfi, 2017). The phasor transform of the linear system (7) for the source $u(t)=\Re\left\{\bar{U} e^{j(\omega t+\phi)}\right\}$, with $\bar{U} \in \mathbb{C}, \omega \in \mathbb{R}$ and $\phi \in \mathbb{R}$, is defined as

$\bar{X} j \omega=A \bar{X}+B \bar{U} e^{j \phi}, \quad \bar{Y}=C \bar{X}$,

where $\bar{X}$ and $\bar{Y}$ are such that $\Re\left\{\bar{X} e^{j \omega t}\right\}$ and $\Re\left\{\bar{Y} e^{j \omega t}\right\}$ are the steadystate responses of $x$ and $y$, respectively.

Definition 5 (Scarciotti \& Astolfi, 2016b). The system (7) and the signal generator (10) are said to be in the real convention if the matrices A, B, $\mathrm{C}, \mathrm{L}$ and $\mathrm{S}$ have real entries. They are said to be in the mixed convention if the matrices $\mathrm{A}, \mathrm{B}, \mathrm{C}$ have real entries and the matrices $\mathrm{L}$ and $\mathrm{S}$ have complex entries.

The following observation, given in Scarciotti and Astolfi (2016b), allows carrying out the phasor analysis of system (7) using a momentdomain approach.

Proposition 1 (Scarciotti \& Astolfi, 2016b). Consider system (7), the input $u(t)=\Re\left\{\bar{U} e^{j(\omega t+\phi)}\right\}$ and its complex-valued realisation (10) with $S=j \omega$, $L=\bar{U} e^{j \phi}$ and $\xi(0)=1$. The phasor transform of system (7), written in the mixed convention, coincides with the Sylvester equation (11) associated to this selection of matrices. The components of $\bar{\Pi}$, which is the unique solution of (11), are the phasors of the state variables of system (7) i.e. $\bar{\Pi}=\bar{X}$.

A drawback of the results of Scarciotti and Astolfi (2016b) is that the matrix $\bar{\Pi}$ is complex-valued. This issue can be solved formulating Proposition 1 using the real convention.

Proposition 2. Consider system (7), the input $u(t)=\Re\left\{\bar{U} e^{j(\omega t+\phi)}\right\}$ and its real-valued realisation (10) with

$S=\left[\begin{array}{cc}0 & \omega \\ -\omega & 0\end{array}\right], L=\left[\begin{array}{l}\mathfrak{R}\left\{\bar{U} e^{j \phi}\right\} \\ \mathfrak{J}\left\{\bar{U} e^{j \phi}\right\}\end{array}\right]^{\top}, \xi(0)=\varepsilon_{2}$. 
Let $\Pi_{R}$ and $\Pi_{I}$ be the first and second column, respectively, of the solution of the Sylvester equation (11) associated to this selection of matrices. Then $\bar{\Pi}=\Pi_{R}+j \Pi_{I}$, where $\bar{\Pi}$ is given in Proposition 1.

Proof. Let $L_{R}=\Re\left\{\bar{U} e^{j \phi}\right\}$ and $L_{I}=\mathfrak{\Im}\left\{\bar{U} e^{j \phi}\right\}$ and write the Sylvester equation (11) partitioning the solution in left and right column, namely

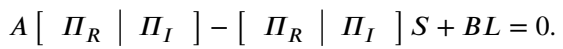

Substituting the values of $S$ and $L$ yields

$\left[A \Pi_{R}+\omega \Pi_{I}+B L_{R} \mid A \Pi_{I}-\omega \Pi_{R}+B L_{I}\right]=0$,

which can be interpreted as a system of two equations. Such a system is solved by

$\Pi_{R}=\left(A^{2}+\omega^{2} \mathbb{I}_{n}\right)^{-1}\left(\omega B L_{I}-A B L_{R}\right)$,

$\Pi_{I}=\left(A^{2}+\omega^{2} \mathbb{I}_{n}\right)^{-1}\left(-\omega B L_{R}-A B L_{I}\right)$.

Consider now the first equation in (12), which can be solved for $\bar{X}$, yielding

$\bar{X}=\left(j \omega \mathbb{I}_{n}-A\right)^{-1} B \bar{U} e^{j \phi}$.

Note now that

$$
\begin{aligned}
\bar{X} & =\left(j \omega \mathbb{I}_{n}-A\right)^{-1}\left(j \omega \mathbb{I}_{n}+A\right)^{-1}\left(j \omega \mathbb{I}_{n}+A\right) B \bar{U} e^{j \phi} \\
& =\left(A^{2}+\omega^{2} \mathbb{I}_{n}\right)^{-1}(-j \omega B-A B) \bar{U} e^{j \phi} \\
& =\left(A^{2}+\omega^{2} \mathbb{I}_{n}\right)^{-1}\left[\left(\omega B L_{I}-A B+L_{R}\right)+j\left(-\omega B L_{R}-A B L_{I}\right)\right]
\end{aligned}
$$

which proves the claim recalling, by Proposition 1 , that $\bar{\Pi}=\bar{X}$.

Definition 6. We call the pair $\left(\Pi_{R}, \Pi_{I}\right)$ phasors in the real convention or simply, with abuse of notation, phasors.

Corollary 2. The phasor of the state $x$ of system (7) is a combination of the moments of the system at $\pm j \omega$, namely $\bar{X}=\Pi_{R}+j \Pi_{I}$. The inverse moment-based phasor transform of the state $x$ of system (7) can be computed as

$x(t)_{s s}=\Pi e^{S t} \xi(0)$,

where $\xi(0)=\varepsilon_{2}$.

Proof. The first claim is a direct consequence of the previous results. To show that (19) is an inverse phasor transform, note that

$$
\begin{aligned}
\Pi e^{S t} \xi(0) & =\left[\Pi_{R} \mid \Pi_{I}\right]\left[\begin{array}{rr}
\cos (\omega t) & \sin (\omega t) \\
-\sin (\omega t) & \cos (\omega t)
\end{array}\right]\left[\begin{array}{l}
1 \\
0
\end{array}\right] \\
& =\Pi_{R} \cos (\omega t)-\Pi_{I} \sin (\omega t) \\
& =\Re\left\{\left(\Pi_{R}+j \Pi_{I}\right)(\cos (\omega t)+j \sin (\omega t))\right\} \\
& =\Re\left\{\bar{X} e^{j \omega t}\right\} . \square
\end{aligned}
$$

This last result is fundamental since it builds a direct relation between the steady-state response of system (7) driven by a particular signal generator (10) and the moment-domain representation of (7).

\section{WEC control problem formulation}

In this study a bottom-referenced spherical heaving point absorber constrained to move in heave only is considered, as illustrated in Fig. 2. The useful energy is converted in the PTO, and can be calculated as the integral of converted power, involving the control force $u(t)$, applied through the PTO system, and the velocity of the device $\dot{x}(t)$ :

$E=\int u(t) \dot{x}(t) d t$

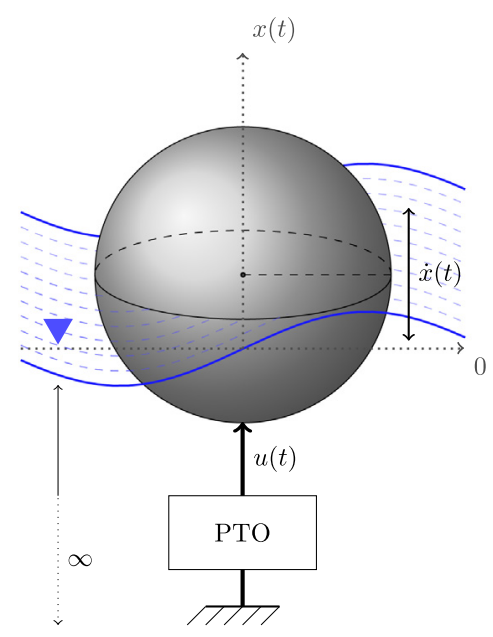

Fig. 2. Wave energy converter.

Consequently, the energy-maximising optimal control formulation computes the PTO force $u(t)$ so that (21) is maximised. Such a calculation is non-trivial mainly due to the irregularity of the poly-chromatic input of the system (the wave excitation force).

\subsection{WEC model}

In this section a brief summary of the modelling of the 1-DOF WEC device (as shown in Fig. 2) is given. The modelling assumptions considered in this section are consistent across a wide variety of WEC energy-maximising model-based optimal control applications presented in the literature, such as, for example, Bacelli and Ringwood (2015), Genest and Ringwood (2016), Li and Belmont (2014), Li, Weiss, Mueller, Townley, and Belmont (2012) and Richter, Magaña, Sawodny, and Brekken (2014) (the reader is referred to Faedo et al. (2017) for a comprehensive list of optimal control strategies that consider similar assumptions).

Considering that the device is referenced from its equilibrium position in an undisturbed wave field and immersed in an infinite-depth sea, the system is subject to fluid-structure interactions which are typically modelled using potential flow theory. The fluid is assumed to be inviscid and incompressible, and the flow is considered irrotational. By applying Newton's second law to the heaving point absorber the following linear hydrodynamic formulation is obtained:

$m \ddot{x}(t)=\mathcal{F}_{r}(t)+\mathcal{F}_{h}(t)+\mathcal{F}_{\text {exc }}(t)-u(t)$,

where $m$ is the mass of the buoy, $x(t)$ the heave excursion, $\mathcal{F}_{\text {exc }}(t)$ the wave excitation force, $\mathcal{F}_{r}(t)$ the radiation force, $\mathcal{F}_{h}(t)$ the hydrostatic restoring force, and $u(t)$ is the control input applied through the PTO system. The hydrostatic force for a floating body is written as $\mathcal{F}_{h}(t)=$ $\rho g V_{i}-\rho g V_{0}$, where $\rho$ is the water density, $g$ is the acceleration due to gravity and $V_{i}=V_{0}-S_{h} x(t)$ represents the immersed volume of the WEC, with $V_{0}$ the immersed volume at the equilibrium position and $-S_{h} x(t)$ a linear approximation of the additional immersed volume depending on the position of the system. The radiation force $\mathcal{F}_{r}(t)$ is also modelled based on linear potential theory and, using the well-known Cummins' equation (Cummins, 1962), is

$\mathcal{F}_{r}(t)=-m_{\infty} \ddot{x}(t)-\int_{0}^{+\infty} \zeta(\tau) \dot{x}(t-\tau) d \tau$,

where $m_{\infty}>0$ represents the added-mass at infinite frequency and $\zeta(t)$ is the (causal) radiation impulse response. Finally, the linearised equation of motion of the WEC is given by

$\left(m+m_{\infty}\right) \ddot{x}(t)+\zeta(t) * \dot{x}(t)+s_{h} x(t)=\mathcal{F}_{e x c}(t)-u(t)$, 
where $s_{h}=\rho g S_{h}>0$ corresponds to the hydrostatic stiffness, and $*$ represents the convolution operator. The equation of motion (24) is of a Volterra integro-differential form, specifically of the convolution class. The internal stability of such an equation, for the WEC case, has been analysed and guaranteed for any physically meaningful values of the parameters and the convolution kernel $\zeta(t)$ involved (Falnes, 2002).

\subsection{Path constraints}

As stated in Section 1, any approach to an optimal control solution for WECs must consider the physical limitations constraining the body's motion and the PTO characteristics. The importance of considering path constrains stems from the fact that the unconstrained solution that maximises energy absorption (Falnes, 2002) is often impossible to achieve, due to unrealistic displacement, velocity and/or force requirements.

Constraints are often considered, for the WEC control problem formulation, on the amplitude (position) $x(t)$, and on the control input (PTO force) $u(t)$, which can be written in a compact form as

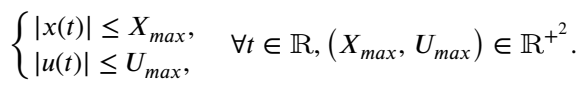

\subsection{Optimal control formulation}

As discussed at the beginning of Section 3.1, the optimal control problem can be informally described as the computation of the PTO force $u(t)$ so that the absorbed energy (21) is maximised. This energymaximising optimal control objective can be achieved by means of a specific objective function, within an optimisation process. This performance objective is strictly related to energy absorption, instead of the traditional "tracking" cost function. The main objective of a wave energy converter is harvesting energy from the incoming wave field, in which the device is immersed. Therefore, the optimal control objective is to maximise the absorbed energy over the time interval $[t, t+T]$, where

$\mathcal{J}=\int_{t}^{t+T} u(\tau) \dot{x}(\tau) d \tau$

while respecting the path constraints defined in (25). Consequently, the optimal control objective can be formulated as,

$$
\max _{u(t)} \mathcal{J}
$$

subject to $\left\{\begin{array}{l}\text { system dynamics (24), } \\ \text { path constraints (25). }\end{array}\right.$

To maximise the absorbed energy, as stated in (27), future knowledge of the motion of the device is required, which only becomes trivial in the case in which the input $\mathcal{F}_{\text {exc }}(t)$ is monochromatic, i.e. it can be represented by $\mathcal{F}_{\text {exc }}(t)=F \cos \left(\omega_{0} t\right)$. This is no longer true in a realistic sea case, in which the excitation force is polychromatic, i.e. it is composed of several harmonics of a fundamental frequency $\omega_{0}$, and prediction of the future values of $\mathcal{F}_{\text {exc }}(t)$ is required within this energy-maximising objective framework. This short-term forecasting requirement is analysed, for example, in Fusco and Ringwood (2012). The optimal control formulation stated in (27) has been solved using different strategies, including diverse modifications on the system dynamics considered, objective function, optimisation method (direct or indirect (Boyd \& Vandenberghe, 2004)) and optimisation algorithms involved (Faedo et al., 2017). In this study, particularly in Section 4, the energy-maximising control problem (27) is formulated and solved in the moment-domain, by applying the moment-based phasor transform to the WEC dynamics specified in (24).

\section{Moment-based WEC control formulation}

In this section the moment-based phasor transform is considered for the WEC energy-maximising optimal control formulation described in (27). Note that the development of the moment-domain theory for linear systems, as described in Section 2.1, is based on a state-space representation approach. Therefore, the WEC dynamics given in (24) are re-written in a more suitable structure, namely

$\dot{x}_{M}(t)=A_{M} x_{M}(t)+B_{M} U(t)$,

$y_{M}(t)=C_{M} x_{M}(t)$,

where $x_{M}(t)=[x(t), \dot{x}(t)]^{\top} \in \mathbb{R}^{n \times 1}$, with $n=2$, is the state-vector of the continuous-time model and $y_{M}(t)=\dot{x}(t) \in \mathbb{R}$ is the output of the system (assuming velocity as measurable output of the device). The function $\mathcal{U}(t)$, assumed to be the input of system (28), is defined as

$\mathcal{U}(t)=\mathcal{F}_{\text {exc }}(t)-u(t)-\zeta(t) * \dot{x}(t)$,

where the actual physical inputs are the excitation force (disturbance) $F_{\text {exc }}(t)$ and the PTO force (control law) $u(t)$. The radiation force convolution term is included as a feedback term, being a pure algebraic manipulation to develop a state-space representation of (24). Under this assumption, the matrices in (28) are given by

$A_{M}=\left[\begin{array}{cc}0 & 1 \\ -\frac{s_{h}}{m+m_{\infty}} & 0\end{array}\right], \quad B_{M}=\left[\begin{array}{c}0 \\ \frac{1}{m+m_{\infty}}\end{array}\right]$,

$C_{M}=\left[\begin{array}{ll}0 & 1\end{array}\right]$.

As a first step, and with the aim of applying the moment-based phasor transform formalism to the WEC optimal control problem, both inputs of (28), $\mathcal{F}_{\text {exc }}(t)$ and $u(t)$, are represented by signal generators, written in implicit form as

$$
\begin{array}{ll}
\dot{\xi}_{\text {exc }}(t)=S \xi_{\text {exc }}(t), & \dot{\xi}_{u}(t)=S \xi_{u}(t), \\
\mathcal{F}_{\text {exc }}(t)=L_{\text {exc }} \xi_{\text {exc }}(t), & u(t)=L_{u} \xi_{u}(t),
\end{array}
$$

where $\xi_{\text {exc }}(t) \in \mathbb{R}^{v \times 1}, \xi_{u}(t) \in \mathbb{R}^{v \times 1}$, with $\xi_{\text {exc }}(0) \neq 0, \xi_{u}(0) \neq 0$ and the matrix $S \in \mathbb{R}^{v \times v}$ is the same for both signal generators, presuming therefore that both inputs can have the same frequency components (with different amplitudes and phases). The pairs $\left(S, L_{\text {exc }}\right)$ and $\left(S, L_{u}\right)$ are assumed to be observable, with $L_{u} \in \mathbb{R}^{1 \times v}$ and $L_{\text {exc }} \in \mathbb{R}^{1 \times v}$. Note that $L_{u}$ and $L_{e x c}$ are the phasors of the control input and the excitation force, respectively. Furthermore, without loss of generality, it is assumed that $\xi_{u}(0)=\xi_{\text {exc }}(0)=\xi(0)=\varepsilon_{v}$.

Since an optimisation procedure is involved in the energymaximising optimal control objective (27), the real convention (Definition 5) is preferred (and adopted) throughout the remainder of this study to apply conventional real-valued optimisation algorithms. Nevertheless, the same results can be easily obtained in the mixed convention, if needed. Using the real convention, the matrix $S$ in (31), can be written in a simple block-diagonal form as

$S=\bigoplus_{p=1}^{k}\left[\begin{array}{cc}0 & \omega_{p} \\ -\omega_{p} & 0\end{array}\right]$,

where $v=2 k$. Then, the steady-state response $y_{s s}(t)$ of system (28) driven by the sum of the outputs of both signal generators in (31) can be computed using a Sylvester equation, see Propositions 1 and 2. Considering superposition, the resulting Sylvester equation for the WEC device case is given by

$A_{M} \Pi_{M}+B_{M}\left(L_{e x c}-L_{u}-\bar{R}\right)=\Pi_{M} S$,

where $\Pi_{M} \in \mathbb{R}^{n \times v}$ and $\bar{R}$ is the moment-domain representation of the radiation convolution term, which is further explained and derived later in this section. Employing the inverse moment-based phasor transform, as defined in (19), the steady-state response can be computed as

$y_{M}(t)_{s s}=C_{M} \Pi_{M} e^{S t} \xi(0)=\bar{V} e^{S t} \xi(0)$, 


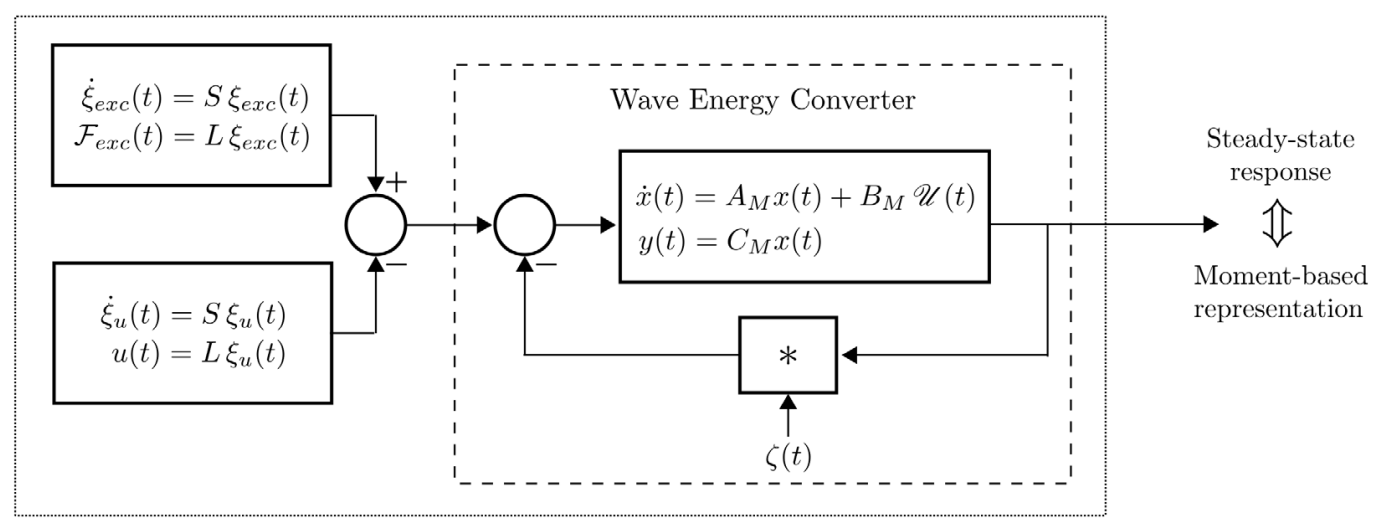

Fig. 3. Block-diagram of the interconnection between system (28) and the signal generator (31).

where $\bar{V}=C_{M} \Pi_{M}$ is the phasor of the output of system (28), i.e. the velocity of the WEC. A schematic of the interconnection between both signal generators in (31) and system (28) is illustrated in Fig. 3.

The objective function (27) depends explicitly on the average power absorbed by the PTO system over a time interval $[t, t+T]$, where $T$ is now defined as $T=2 \pi / \omega_{0}$, and denotes the fundamental period. Likewise, $\omega_{0}$ represents the fundamental frequency. As proven in Scarciotti and Astolfi (2016b), the moment-based phasor transform maintains the physical meaning of the original variables, which is of paramount importance for the successful application of such a mathematical tool in the WEC control formulation. Consequently, the instantaneous power in steady-state can be computed using the inverse moment-based phasor transform (19) as

$p(t)_{s s}=\dot{x}_{M}(t)_{s s} u(t)=\left(\bar{V} e^{S t} \varepsilon_{v}\right)\left(L_{u} e^{S t} \varepsilon_{v}\right)$.

One usual assumption for the numerical generation of the wave excitation force $\mathcal{F}_{\text {exc }}(t)$ in many ocean engineering applications is that it can be expressed as the sum of $k$ harmonics of the fundamental frequency $\omega_{0}$ (Mérigaud \& Ringwood, 2017). The following proposition allows the computation of the average power as a simple vector product, by further exploiting the properties of the moment-based characterisation.

Proposition 3. Consider the expression for the instantaneous power (35) and the signal generators (31). Define the constant values of $S$ in (32) as $\omega_{p}=p \omega_{0}, \forall p=1, \ldots, k$ with $k \geq 1$ integer (i.e. $k$ harmonics of the fundamental frequency $\omega_{0}$ ). Then the average power $P_{s s}$ absorbed over the time period $[t, t+T]$, where $T=2 \pi / \omega_{0}$, can be computed using the momentbased phasor transform as

$P_{s s}=\frac{1}{2} \bar{V} L_{u}^{\top}$

Proof. The average power over the time period $[t, t+T]$ can be expressed as

$$
\begin{aligned}
P_{S S} & =\frac{1}{T} \int_{t}^{t+T}\left(\bar{V} e^{S \tau} \varepsilon_{v}\right)\left(L_{u} e^{S \tau} \varepsilon_{v}\right) d \tau \\
& =\bar{V}\left[\frac{1}{T} \int_{t}^{t+T}\left(e^{S \tau} \varepsilon_{v}\right)\left(e^{S \tau} \varepsilon_{v}\right)^{\top} d \tau\right] L_{u}^{\top} .
\end{aligned}
$$

Considering that the vector $\left(e^{S t} \varepsilon_{v}\right) \in \mathbb{R}^{\nu \times 1}$ can be conveniently written as

$$
e^{S t} \varepsilon_{\nu}=\left[\begin{array}{c}
\cos \left(\omega_{0} t\right) \\
-\sin \left(\omega_{0} t\right) \\
\vdots \\
\cos \left(k \omega_{0} t\right) \\
-\sin \left(k \omega_{0} t\right)
\end{array}\right]=\left[\begin{array}{c}
\psi_{\omega_{0}}^{+}(t) \\
\psi_{\omega_{0}}^{-}(t) \\
\vdots \\
\psi_{k \omega_{0}}^{+}(t) \\
\psi_{k \omega_{0}}^{-}(t)
\end{array}\right],
$$

the matrix $\left(e^{S t} \varepsilon_{v}\right)\left(e^{S t} \varepsilon_{v}\right)^{\top}$ is symmetric, with elements given by the expressions ${ }^{1}$

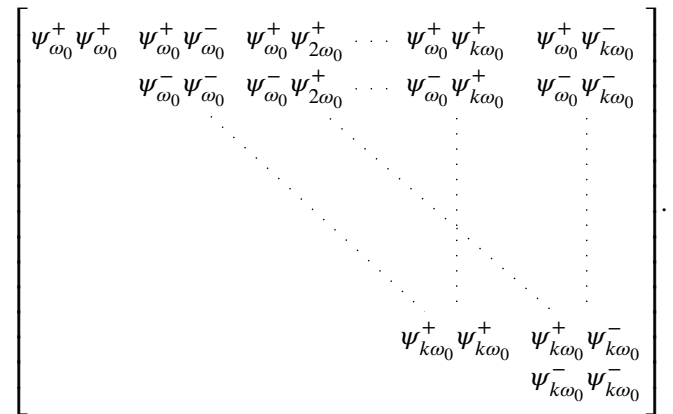

Note that $\psi_{p \omega_{0}}^{+}(t), \psi_{p \omega_{0}}^{-}(t) \in L^{2}(\mathbb{R})[t, t+T], \forall p=1, \ldots, k$, and that the set of functions $\left\{\psi_{p \omega_{0}}^{+}(t), \psi_{p \omega_{0}}^{-}(t)\right\}_{p=1}^{k}$ is orthogonal under the inner product defined in (1), i.e.,

$$
\begin{aligned}
& \left\langle\psi_{p \omega_{0}}^{+}(t), \psi_{p \omega_{0}}^{+}(t)\right\rangle=\frac{1}{2} T,\left\langle\psi_{p \omega_{0}}^{-}(t), \psi_{p \omega_{0}}^{-}(t)\right\rangle=\frac{1}{2} T, \\
& \left\langle\psi_{p \omega_{0}}^{+}(t), \psi_{p \omega_{0}}^{-}(t)\right\rangle=0, \quad\left\langle\psi_{p \omega_{0}}^{-}(t), \psi_{p \omega_{0}}^{+}(t)\right\rangle=0, \\
& \left\langle\psi_{p \omega_{0}}^{\circ}(t), \psi_{q \omega_{0}}^{\circ}(t)\right\rangle=0, \quad \forall p \neq q \text { and any } \circ=\{+,-\} .
\end{aligned}
$$

By noting that the resulting operations in the integral term of (37) are merely inner products between functions of the set $\left\{\psi_{p \omega_{0}}^{+}(t), \psi_{p \omega_{0}}^{-}(t)\right\}_{p=1}^{k}$, scaled by $1 / T$, the equality

$\frac{1}{T} \int_{t}^{t+T}\left(e^{S \tau} \varepsilon_{v}\right)\left(e^{S \tau} \varepsilon_{v}\right)^{\top} d \tau=\frac{1}{2} \mathbb{I}_{v}$

holds, and the average power in (37) can be computed as $P_{s s}=$ $\frac{1}{2} \bar{V} \mathbb{I}_{\nu} L_{u}^{\top}=\frac{1}{2} \bar{V} L_{u}^{\top}$, which concludes the proof.

By Proposition 3, considering that the dynamics of system (28) under the moment-phasor transform are given by the Sylvester equation in (33), the equality constrained optimal control problem over the time period $[t, t+T]$ defined in (27), without path constraints (which are discussed and included later in Section 4.1), can be written in the moment-domain as

$\max _{L_{u}} \frac{1}{2} \bar{V} L_{u}^{\top}$

subject to:

$A_{M} \Pi_{M}+B_{M}\left(L_{e x c}-L_{u}-\bar{R}\right)=\Pi_{M} S$,

$C \Pi_{M}=\bar{V}$.

The optimisation problem in (42) is a mixed state-input equalityconstrained problem. Moreover, it is not possible to develop any

\footnotetext{
${ }^{1}$ Only the elements of the main diagonal, and those above, are shown in (39), for simplicity of notation. The dependence on $t$ is also dropped.
} 
straightforward conclusion regarding the existence and uniqueness of the optimal solution. In the following, and before going further with the analysis of (42), the convolution term in $\mathscr{U}$ in (29) is expressed analytically by its moment-domain equivalent $\bar{R}$. By recalling Eq. (34), the convolution integral (in steady-state) can be written as

$\int_{0}^{+\infty} \zeta(\tau) \dot{x}(t-\tau) d \tau=\bar{V} \int_{0}^{+\infty} \zeta(\tau)\left(e^{S(t-\tau)} \varepsilon_{\nu}\right) d \tau$,

where the vector $e^{S(t-\tau)} \varepsilon_{v}$ can be expanded as in (38). Then, the convolution integral can be written in vector form as

$\bar{V}\left[\begin{array}{c}\zeta(t) * \psi_{\omega_{0}}^{+}(t) \\ \zeta(t) * \psi_{\omega_{0}}^{-}(t) \\ \vdots \\ \zeta(t) * \psi_{k \omega_{0}}^{+}(t) \\ \zeta(t) * \psi_{k \omega_{0}}^{-}(t)\end{array}\right]$.

Considering the elements $\psi_{q \omega_{0}}^{+}(t), \psi_{q \omega_{0}}^{-}(t)$, it is possible to recognise that expression (44) depends on two general convolution operations, namely, $\zeta(t) * \psi_{q \omega_{0}}^{+}(t)$ and $\zeta(t) * \psi_{q \omega_{0}}^{-}(t)$. Expanding the first expression, using well-known trigonometric identities, yields

$$
\begin{aligned}
\zeta(t) * \psi_{q \omega_{0}}^{+}(t)= & \cos \left(q \omega_{0} t\right) \int_{0}^{+\infty} \zeta(t) \cos \left(q \omega_{0} t\right) d t+ \\
& \sin \left(q \omega_{0} t\right) \int_{0}^{+\infty} \zeta(t) \sin \left(q \omega_{0} t\right) d t .
\end{aligned}
$$

The integral operations involved in (45) are well-known physical relations within the hydrodynamic community. In fact, by considering Ogilvie's frequency domain relations (Ogilvie, 1964), the trigonometric integral terms can be evaluated explicitly as:

$$
\begin{aligned}
& \int_{0}^{+\infty} \zeta(t) \cos \left(q \omega_{0} t\right) d t=\mathcal{R}\left(q \omega_{0}\right)=r_{q \omega_{0}}, \\
& \int_{0}^{+\infty} \zeta(t) \sin \left(q \omega_{0} t\right) d t=-q \omega_{0}\left(\mathcal{M}\left(q \omega_{0}\right)-m_{\infty}\right)=m_{q \omega_{0}},
\end{aligned}
$$

where $\mathcal{R}(\omega)$ is the frequency-dependent radiation resistance and $\mathcal{M}(\omega)$ is the frequency-dependent added-mass of the heaving body WEC considered (the reader is referred to Falnes, 2002 for further information). By performing similar operations on $\zeta(t) * \psi_{q \omega_{0}}^{-}(t)$ yields

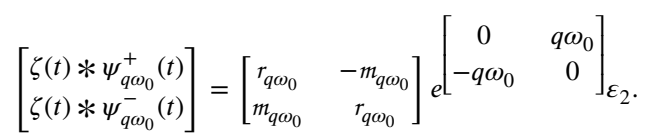

Finally, considering all the elements in expression (44), the momentdomain equivalent of the convolution integral can be computed as

$\zeta(t) * \dot{x}(t)=(\bar{V} \mathscr{R}) e^{S t} \varepsilon_{v}=\bar{R} e^{S t} \varepsilon_{v}$,

where $\mathscr{R}$ is a block-diagonal matrix defined by

$\mathscr{R}=\bigoplus_{p=1}^{k}\left[\begin{array}{cc}r_{p \omega_{0}} & -m_{p \omega_{0}} \\ m_{p \omega_{0}} & r_{p \omega_{0}}\end{array}\right]$

and the values involved depend on the added-mass and the radiation resistance of the device at each specific frequency considered.

To obtain further insight on the optimisation formulation of (42), Eq. (33) is analysed using a linear geometric approach. For convenience, (33) is re-written as

$A_{M} \Pi_{M}+\Pi_{M}(-S)=-B_{M}\left(L_{e x c}-L_{u}-\bar{R}\right)$.

From a geometric perspective, (50) can be seen as a linear endomorphism $\Gamma$, i.e.

$$
\begin{aligned}
& \Gamma: \mathbb{R}^{n \times v} \longrightarrow \mathbb{R}^{n \times v}, \quad \Pi_{M} \in \mathbb{R}^{n \times v}, \\
& \Gamma\left\{\Pi_{M}\right\} \mapsto A_{M} \Pi_{M}+\Pi_{M}(-S),
\end{aligned}
$$

and the matrix $-B_{M}\left(L_{\text {exc }}-L_{u}-\bar{R}\right) \in \operatorname{Im}\{\Gamma\} \subset \mathbb{R}^{n \times v}$. Considering an ordered canonical basis for $\mathbb{R}^{n \times v}$, in accordance with the vec operator (see Definition 2), the elements of $\Pi_{M}$ in (51) can be computed as ${ }^{2}$ (Van Loan, 2000)

$\operatorname{vec}\left\{\Pi_{M}\right\}=\left(S \hat{\oplus} A_{M}\right)^{-1} \operatorname{vec}\left\{-B_{M}\left(L_{e x c}-L_{u}-\bar{R}\right)\right\}$,

where the existence of $\left(S \hat{\oplus} A_{M}\right)^{-1}$ is guaranteed by Corollary 1 , since $\sigma\left(A_{M}\right) \cap \sigma(S)=\emptyset$ for any realistic device parameters involved in the matrix $A_{M}$ and, therefore, $\Gamma$ is an automorphism, i.e. $\operatorname{Ker}\{\Gamma\}=\{0\}$. Using the vec operator equivalence stated in Property 1 , and recalling that $\bar{V}=C_{M} \Pi_{M}$, the solution of the Sylvester equation, derived in (52), is given by

$\operatorname{vec}\{\bar{V}\}=\Gamma_{*}\left(\operatorname{vec}\left\{L_{e x c}\right\}-\operatorname{vec}\left\{L_{u}\right\}-\operatorname{vec}\{\bar{R}\}\right)$,

where the matrix $\Gamma_{*} \in \mathbb{R}^{n v \times n v}$ is given by

$\Gamma_{*}=\left(\mathbb{I}_{\nu} \otimes C_{M}\right)\left(S \hat{\oplus} A_{M}\right)^{-1}\left(\mathbb{I}_{\nu} \otimes-B_{M}\right)$.

Substituting the moment-domain equivalent of the radiation convolution term obtained in (48), and after algebraic manipulations, the expression obtained in (53) can be written as

$\operatorname{vec}\{\bar{V}\}=\left(\mathbb{I}_{v}+\Gamma_{*} \mathscr{R}^{\mathrm{T}}\right)^{-1} \Gamma_{*}\left(\operatorname{vec}\left\{L_{e x c}\right\}-\operatorname{vec}\left\{L_{u}\right\}\right)$.

Finally, by recalling that the basis considered for the computation of the matrix involved in (52) is canonical, the coordinates of the phasors, and the phasors themselves, are related by a simple transposition operation, i.e. $\operatorname{vec}\{\bar{V}\}=\bar{V}^{\top}, \operatorname{vec}\left\{L_{e x c}\right\}=L_{e x c}^{\top}$, and $\operatorname{vec}\left\{L_{u}\right\}=L_{u}^{\top}$. Consequently, $\bar{V}$ can be computed as

$\bar{V}=\left(L_{e x c}-L_{u}\right) \Gamma_{*}^{\mathscr{R}}$,

where $\Gamma_{*}^{\mathscr{R}} \in \mathbb{R}^{v \times v}$ is given by

$\Gamma_{*}^{\mathscr{R}}=\left[\left(\mathbb{I}_{v}+\Gamma_{*} \mathscr{R}^{\top}\right)^{-1} \Gamma_{*}\right]^{\top}$.

By (56) the equation of motion (50) can be explicitly solved with respect to the phasor of the velocity $\bar{V}$. Substituting $\bar{V}$ into the optimal control formulation (42), the energy-maximising controller can be designed by maximising the absorbed energy as

$\max _{L_{u}}-\frac{1}{2} L_{u} \Gamma_{*}^{\mathscr{R}} L_{u}^{\top}+\frac{1}{2} L_{\text {exc }} \Gamma_{*}^{\mathscr{R}} L_{u}^{\top}$,

which represents a quadratic problem (QP) involving only $L_{u}$. The phasors of the state variables have been eliminated by substitution and the optimisation is now carried out over the control variable phasor $L_{u}$ only. The equality constrained mixed state-input formulation (42) has been transformed into an unconstrained quadratic program.

In the following, an important result regarding the concavity of the quadratic program involved in the moment-domain control formulation defined in (58) is derived. Noting that the structure of $\Gamma_{*}$ is blockdiagonal, and considering Property 2, it is possible to state and prove the following proposition.

Proposition 4. The QP formulation in (58) is strictly concave for any physically meaningful values of the system parameters in (30).

Proof. Firstly, and as a consequence of Property 2, the QP formulation (58) is strictly concave if and only if (Boyd \& Vandenberghe, 2004) $\mathscr{H}\left\{-\Gamma_{*}^{\mathscr{R}}\right\}$ is negative-definite, which is equivalent to the condition that $\mathscr{H}\left\{\Gamma_{*}^{\mathscr{R}}\right\}$ has to be positive-definite. Note that, since both $\Gamma_{*}$ in (54) and $\mathscr{R}$ in (49) are block-diagonal matrices, the structure of $\Gamma_{*}^{*}$ is indeed block-diagonal. Explicitly,

$$
\begin{aligned}
\Gamma_{*}^{\mathscr{R}} & =\bigoplus_{p=1}^{k} \frac{1}{\beta}\left[\begin{array}{cc}
\left(p \omega_{0}\right)^{2} r_{p \omega_{0}} & -\alpha \\
\alpha & \left(p \omega_{0}\right)^{2} r_{p \omega_{0}}
\end{array}\right], \\
\alpha & =\left(p \omega_{0}\right)\left[\left(m+m_{\infty}\right)\left(p \omega_{0}\right)^{2}+m_{p \omega_{0}}\left(p \omega_{0}\right)-s_{h}\right], \\
\beta & =\left(p \omega_{0}\right)^{2} r_{p \omega_{0}}^{2}+\left(\alpha /\left(p \omega_{o}\right)\right)^{2},
\end{aligned}
$$

\footnotetext{
${ }^{2}$ Note also that $S$ is skew-symmetric.
} 
and finally,

$\mathscr{H}\left\{\Gamma_{*}^{\mathscr{R}}\right\}=\bigoplus_{p=1}^{k} \frac{1}{\beta}\left[\begin{array}{cc}\left(p \omega_{0}\right)^{2} r_{p \omega_{0}} & 0 \\ 0 & \left(p \omega_{0}\right)^{2} r_{p \omega_{0}}\end{array}\right]>0$.

The result provided by Proposition 4 is important for the energymaximising application: the unconstrained moment-domain optimal control formulation for the WEC device (58) has always a unique (global) maximum, allowing the utilisation of well-known and efficient quadratic programming solvers (Boyd \& Vandenberghe, 2004) to compute the optimal control law in real-time. Furthermore, this momentdomain formulation allows the inclusion of constrains on the motion of the device as demonstrated in Section 4.1.

\subsection{Force and amplitude constraints}

As discussed in Section 3.2, constraints on the control input and the oscillation amplitude reflect physical limitations on the device or its components. To ensure the feasibility of the control input computed by the optimal control formulation (58) and to secure the durability of the device in real sea conditions, constraints are considered in the momentdomain framework as follows. Recall the inequality constraints describing the maximum allowed force and maximum allowed oscillation amplitude in (25). Using the inverse moment-based phasor transform (19) the mappings

$\left\{\begin{array}{l}|u(t)| \leq U_{\max }, \\ |x(t)| \leq X_{\max },\end{array} \quad \rightarrow \quad\left\{\begin{array}{l}\left|L_{u} e^{S t} \varepsilon_{v}\right| \leq U_{\max }, \\ \left|\bar{X} e^{S t} \varepsilon_{v}\right| \leq X_{\max },\end{array}\right.\right.$

where $\bar{X}$ represents the phasor of the position $x(t)$, hold. Before going further with the development of (61), the following property of the moment-based phasor transform is recalled from Scarciotti and Astolfi (2016b).

Proposition 5 (Scarciotti \& Astolfi, 2016b). Consider a dynamical system given by the differential equation

$\dot{x}(t)=u(t)$.

Then, when applying the moment-based phasor transform, the phasor of $\dot{x}(t)$ is $X S$, where $X$ is the phasor of $x(t)$. In a similar way, the moment-domain equivalent of $\int x(\tau) d \tau$ is given by $X S^{-1}$.

Using Proposition 5 it is possible to re-write (61) as

$\left\{\begin{array}{l}\left|L_{u} e^{S t} \varepsilon_{v}\right| \leq U_{\max }, \\ \left|\bar{V} S^{-1} e^{S t} \varepsilon_{v}\right| \leq X_{\max },\end{array}\right.$

where $\bar{V}=C_{M} \Pi_{M}$ is the phasor of the velocity. Inspired by spectral direct transcription techniques (as applied, for example, in Bacelli and Ringwood (2015)), one possible approach to deal with the constraints (63) is to enforce them only at a set of specified time instants (collocation points), i.e. $t_{1}, \ldots, t_{N_{c}}$. Defining the vectors $\Lambda \in \mathbb{R}^{\nu \times N_{c}}$ and $\Delta \in \mathbb{R}^{\nu \times 2 N_{c}}$ as

$\Lambda=\left[\begin{array}{lll}e^{S t_{0}} \varepsilon_{\nu} & \ldots & e^{S t_{N_{c}}} \varepsilon_{v}\end{array}\right] \quad \Delta=\left[\begin{array}{ll}\Lambda & -\Lambda\end{array}\right]$

and substituting $\bar{V}$ using (56), the constraints in (63), at the collocation points, can be written as a set of linear inequalities given by

$L_{u} \Delta \leq U_{\max } \mathbb{1}_{1 \times 2 N_{c}}$,

$L_{u}\left(-\Gamma_{*}^{\mathscr{R}}\right) S^{-1} \Delta \leq X_{\max } \mathbb{1}_{1 \times 2 N_{c}}-L_{e x c} \Gamma_{*}^{\mathscr{R}} S^{-1} \Delta$.

Finally, the inequality constrained QP optimal control formulation can be written as

$\max _{L_{u}}-\frac{1}{2} L_{u} \Gamma_{*}^{\mathscr{R}} L_{u}^{\top}+\frac{1}{2} L_{e x c} \Gamma_{*}^{\mathscr{R}} L_{u}^{\top}$,

subject to:

$L_{u} \Delta \leq U_{\max } \mathbb{1}_{1 \times 2 N_{c}}$,

$L_{u}\left(-\Gamma_{*}^{\mathscr{R}}\right) S^{-1} \Delta \leq X_{\max } \mathbb{1}_{1 \times 2 N_{c}}-L_{e x c} \Gamma_{*}^{\mathscr{R}} S^{-1} \Delta$, where the uniqueness of the global maximum, for the unconstrained case, is guaranteed by Proposition 4. Eq. (66) explicitly shows the potential of this moment-domain formulation: the energy-maximising optimal control problem (27) can be transcribed using moments into an inequality constrained convex $Q P$ which, as discussed and illustrated in Section 5, allows the computation of an optimal control law in realtime (using efficient state-of-the-art QP solvers Boyd \& Vandenberghe, 2004) that maximises the energy absorption from waves and respects the physical limitations of both the device and the PTO system.

\section{Application to a heaving point absorber WEC}

In this section, a heaving point absorber WEC (as illustrated in Fig. 2) is considered. The radius of the device is chosen as $5[\mathrm{~m}]$. Results are presented for both regular (monochromatic) and irregular (polychromatic) waves. It is useful to first evaluate the control strategy by considering the simple case of regular waves (although not a realistic case), since some numerical results can be contrasted and compared, to some extent, with well-known analytical statements.

This section demonstrates that the control solution in (66) can be solved efficiently in real-time for a typical WEC, respecting constrains on the WEC motion and PTO force. In addition, we introduce a smoothness metric on the control signal to give a handle on control aggressiveness (which is directly linked to WEC operational costs Chang, Jones, Roberts, \& Neary, 2018; Nielsen, Pedersen, Andersen, \& Ambhl, 2017), which is a major cost driver for wave energy systems.

\subsection{Regular waves}

The following case is adopted as "nominal" and is considered repeatedly in the subsequent analysis performed for the regular wave case:

- wave height $=3[\mathrm{~m}]$.

- wave period $T=8[\mathrm{~s}]$.

- maximum device displacement $X_{\max }=2[\mathrm{~m}]$.

- maximum PTO force $U_{\max }=0.4 \times 10^{6}[\mathrm{~N}]$.

Deviations from the nominal case are considered (and detailed) when necessary.

In the regular wave case the excitation force is considered to be monochromatic, i.e. $\mathcal{F}_{\text {exc }}=F \cos \left(\omega_{0}\right)$, where $\omega_{0}$ is the fundamental frequency. Although the actual input is composed of only one frequency, the optimal control input $u(t)$ can contain higher frequency components, since its calculation comes from a constrained optimal control formulation (Boyd \& Vandenberghe, 2004). The selection of the number of frequency harmonics $k$ involved in the dynamics of each signal generator, i.e. the matrix $S$, implies an immediate trade-off between three important quantities:

1. computational time required by the optimisation process,

2. absorbed energy over the period considered,

3. rate of change of the control input.

For such a trade-off analysis different values of $k$ are considered in simulation, namely $k \in\{2,3,5,10,15,20,40\}$. The computational time is not comprehensively analysed in this study, since the computation of the nominal case of interest, for all the values of $k$ considered, can be done in less than one second (implemented in MAтLAB). This is consistent with the typical sampling rate of a full-scale WEC, which would have a dominant time constant of around 10 seconds. Moreover, we note that this algorithm has been implemented in MATLAB which is an interpreted language: the computational time can be considerably improved by implementing this algorithm in any compiled language, such as $\mathrm{C}$ or $\mathrm{C}++$. In the case of the absorbed energy as a function of the number of frequencies considered, simulation results for different wave periods can be seen in Fig. 4. In particular, Fig. 4 illustrates the ratio 
of absorbed energy, defined as $E_{k} / E_{40}$, against each frequency $k . E_{40}$ represents the absorbed energy when 40 harmonics are considered for the signal generator, i.e. $k=40$, and this is assumed as the maximum required number of frequencies, since an almost imperceptible change in energy absorption can be observed after 40 frequencies. It can be appreciated that, after $10-15$ frequencies, $E_{k} / E_{40}$ is indeed approximately unity, indicating that little improvement can be obtained by considering more than 10-15 harmonics. Finally, the rate of change of the control input $\dot{u}(t)$ is analysed as an important trade-off, since highvalues of $\dot{u}(t)$ imply the necessity of a fast PTO response to implement the optimal control profile obtained by the optimisation process. To perform such an analysis two different indicators are considered: the maximum absolute value of the rate of change of the control input, i.e. $|\dot{u}(t)|_{k}^{\max }$ and a "smoothness" measure, defined later in this section. In Fig. $5,|\dot{u}(t)|_{k}^{\max }$ is shown for different values of the PTO constraints. In particular, the set of constraints chosen for such a simulation is $U_{\max } \in$ $\{0.7,0.6,0.2,0.1\} \times 10^{6}[\mathrm{~N}]$. Essentially, the value of $|\dot{u}(t)|_{k}^{\max }$ provides a measure of the maximum "speed" required from the PTO mechanism to achieve the optimal control profile computed. Analysing Fig. 5, it can be acknowledged that a tighter constraint $U_{\max }$ generates lower values for the rate of change of $u(t)$ and, hence, both the maximum force and speed required from the actuator are of lower magnitude. Nevertheless, the maximum value of $|\dot{u}(t)|$ does not provide a concise measure of the "smoothness" of the optimal profile computed, but rather a value of the maximum rate of change, which can occur at isolated time instants. An overall estimate of the smoothness or "fairness" (Meier \& Nowacki, 1987) of the optimal control profile, calculated by the optimisation process proposed, can be given in terms of a well-known differential geometry concept: the curvature. Geometrically, the curvature at a point on a curve is defined to measure how quickly the curve changes direction at that point. Analysing $u(t)$ as a smooth parametric curve, and based on the preceding concept of curvature, the following definition for a smoothness parameter is proposed:

$S_{k}^{U_{\max }}=\int_{t}^{t+T}\left|\frac{d^{2} u_{k}^{U_{\max }}(t)}{d t^{2}}\right|^{2} d t$,

where $u_{k}^{U_{\max }}(t)$ denotes the optimal control input computed using $k$ frequencies, constrained to the value $U_{\max }$. The integrand in (67) is proportional to the curvature, and its basically used as an approximation of the real value. A similar "smoothness" measure is used for several interpolation approaches, such as the smoothing spline method (Reinsch, 1967). The higher the value of $S_{k}^{U_{\max }}$, the more quickly $\dot{u}(t)$ changes direction on $[t, t+T]$ which, in the case of this application, implies a fast PTO response during the whole period. Consequently, it is expected that the values of $S_{k}^{U_{\max }}$ scales naturally with the number of frequencies considered for the input, demanding a quicker response from the actuator during the time period analysed. This can be further appreciated in Fig. 6, where the parameter $S_{k}^{U_{\max }}$ is considered for $k \in\{2,3,5,10,15,20,40\}$. Fig. 6 also illustrates the effect of tighter constraints on the maximum force for the PTO system: a tighter constraint value requires less "influence" of the high-frequency components, leading to a much lower value of $S_{k}^{U_{\max }}$ in low values of $k$ and, hence, a smoother profile.

This simple trade-off analysis allows a proper use of the additional degree of freedom $k$, as a function of the total absorbed energy and the response of the actual PTO system involved in the application. In this case study, combining and analysing the results obtained for the absorbed energy and overall smoothness of the control input computed for all the proposed values of $k, 10$ frequencies are retained for all the subsequent regular wave simulations, since such a value of $k$ yields reasonable results for both absorbed energy and overall smoothness of the optimal control law.

For a more comprehensive analysis of the motion of the device under monochromatic excitation, two cases are simulated: the nominal case, as described at the beginning of this section and the same case but in which only the maximum displacement (amplitude) is constrained

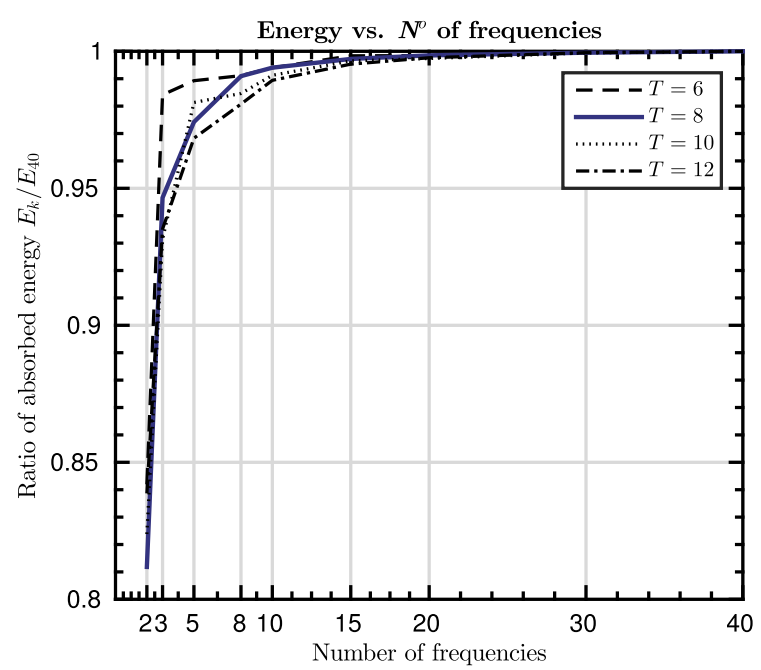

Fig. 4. Ratio of absorbed energy $E_{k} / E_{40}$ for different values of $k$ and different wave periods (nominal case in thin-blue line).

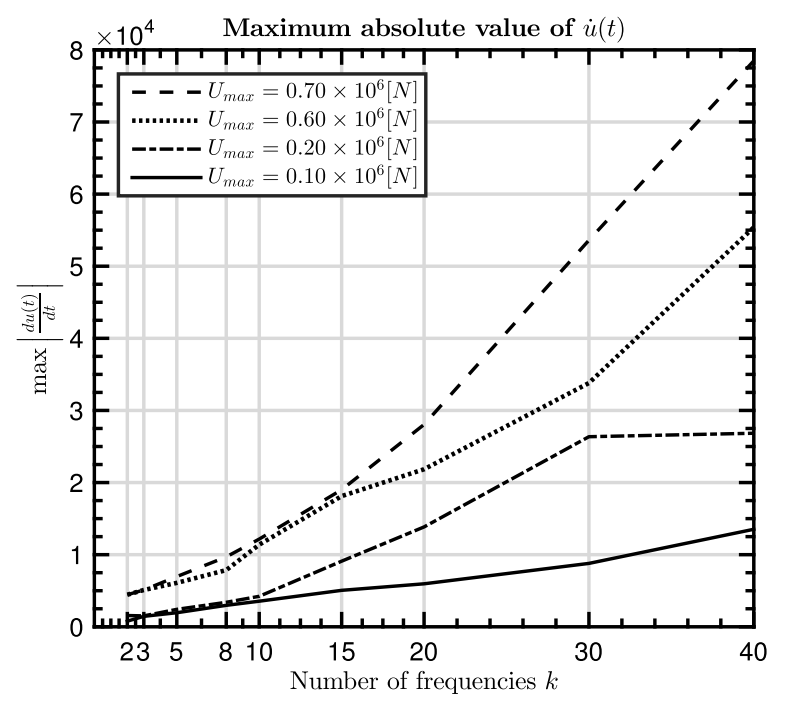

Fig. 5. Maximum absolute value for the rate of change of $u(t)$.

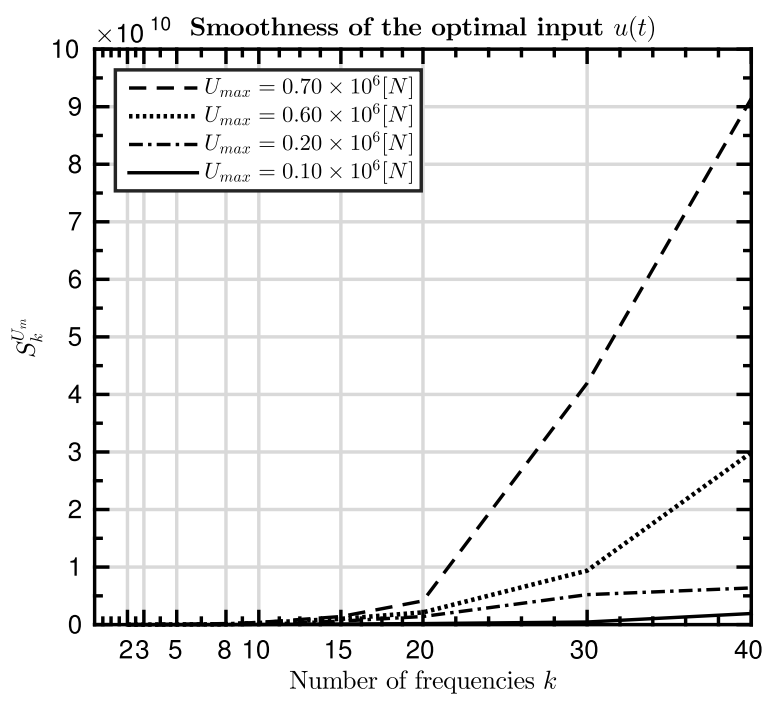

Fig. 6. Measure of smoothness of the computed optimal control PTO force as a function of the number $k$ of frequencies considered. 


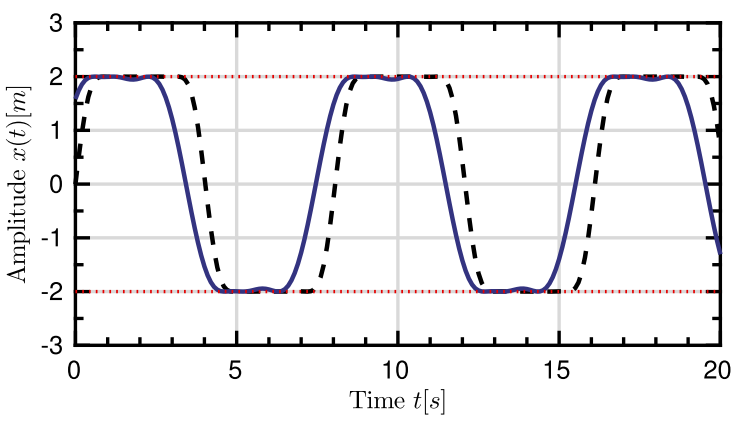

(a)

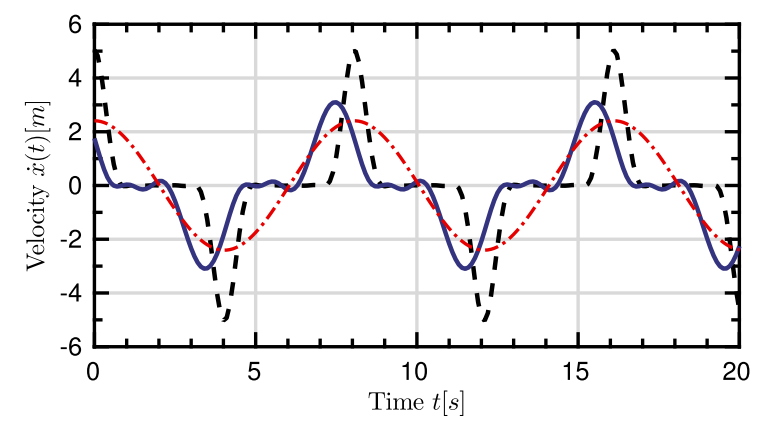

(b)

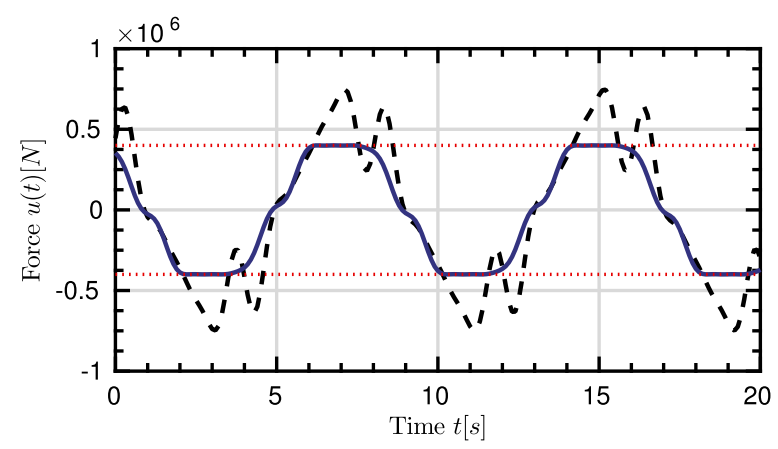

(c)

Fig. 7. Amplitude (a), velocity (b) and optimal PTO force (c) for the nominal regular waves case (solid-blue) and the case where only the amplitude is constrained (dashed-black). In (b), the excitation force is also presented (scaled, dotted-red), showing the "in-phase" maximum power absorption condition when only the amplitude is constrained.

with a value of $X_{\max }=2[\mathrm{~m}]$. Results on the amplitude, velocity and optimal control input for both cases are given in Figs. 7(a), 7(b) and 7(c), respectively. As a first result it can be acknowledged that both constraints, namely $X_{\max }$ and $U_{\max }$, are consistently respected. In the nominal case the control input is constrained to a value of $0.4 \times 10^{6}[\mathrm{~N}]$, which represents approximately half of the maximum value that takes place when only the amplitude is constrained, as seen in Fig. 7(c). Regarding the velocity of the device, it can be appreciated that, for the case where only the amplitude is constrained, the velocity remains "in phase" with the excitation force, agreeing with the well-known result in the case of (fully) unconstrained maximum power absorption (Falnes, 2002). The term "in phase" is used here to denote that the peaks (maxima and minima) of both signals occur at approximately the same time instant since, essentially, the formal concept of phase is no longer defined for signals containing multiple frequencies. Such a behaviour does not hold any more for the case when both the amplitude and the PTO force are constrained at the same time, as can be observed from
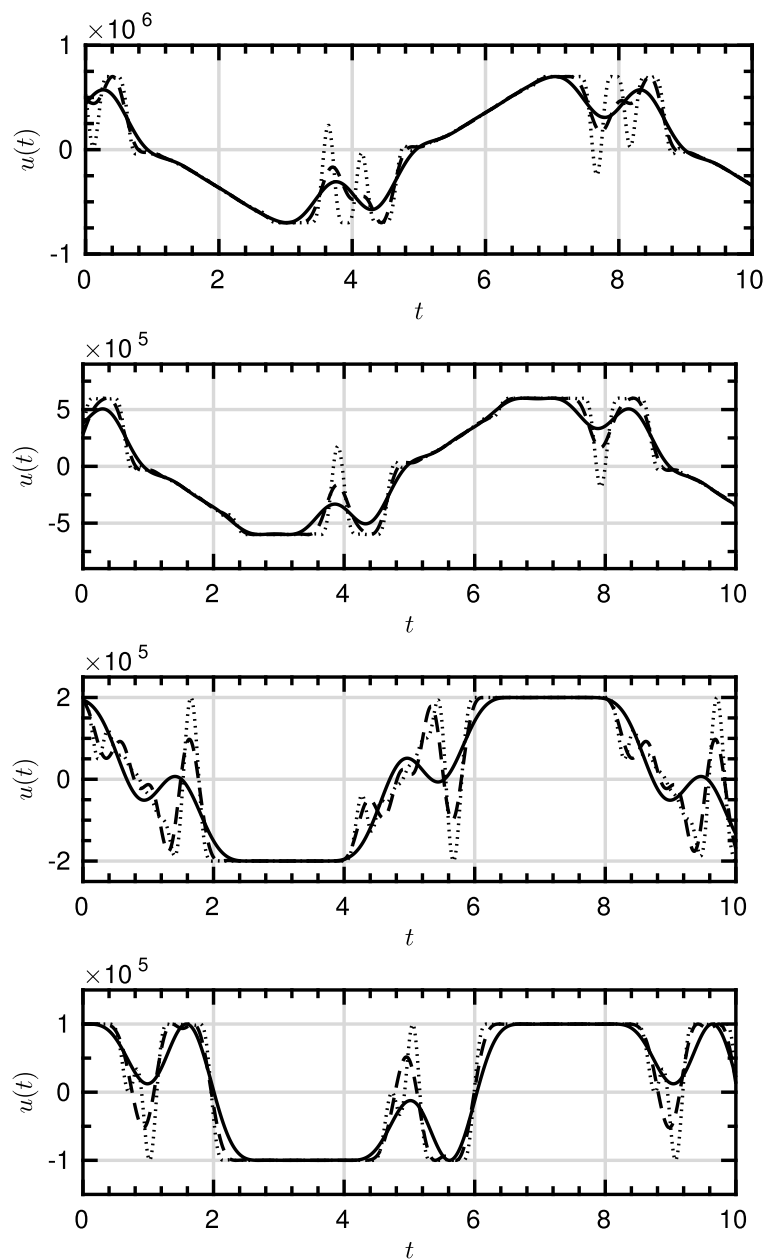

Fig. 8. Optimal control profile for several values of constraints. From top to bottom: $U_{\max }=\{0.7,0.6,0.2,0.1\} \times 10^{6}[\mathrm{~N}]$ and frequency components $k=10$ (solid), $k=20$ (dashed), $k=40$ (dotted).

Fig. 7(b). Finally, in Fig. 8, the set of constraints used for the trade-off analysis earlier in this section, i.e. $U_{\max }=\{0.7,0.6,0.2,0.1\} \times 10^{6}[\mathrm{~N}]$, is considered, together with an amplitude constraint of $X_{\max }=2[\mathrm{~m}]$, while varying the number $k$ of frequency harmonics. It can be noted how the signal becomes "smoother" when tightening the constraint value on the PTO force, consistently with the results obtained in Figs. 5 and 6.

\subsection{Irregular waves}

For the irregular waves case a Joint North Sea Wave Project (JONSWAP) (Hasselmann, 1973) spectrum is considered, with a peak period $T_{p}=10[\mathrm{~s}]$ and a significant wave height $H_{s}=3[\mathrm{~m}]$. In this case the fundamental frequency $\omega_{0}$ and the number of harmonics $k$ have to be chosen so that the most energy-significant components of the excitation force are included in the corresponding signal generator. This could be done by simply looking at the spectrum of $\mathcal{F}_{\text {exc }}$. Of course, consistently with the regular wave case, a trade-off emerges between $k$, the computational time required by the optimisation problem, and the smoothness of the profile obtained. Taking into account the spectrum considered and performing an analysis on the absorbed power and the smoothness of the optimal input profile, similar to the monochromatic input case (Section 5.1), the fundamental frequency is chosen as $\omega_{0}=$ $0.1[\mathrm{rad} / \mathrm{s}]$ and the number of frequency harmonics is chosen as $k=30$.

Simulation results for the motion of the device, i.e. amplitude and velocity, are presented in Figs. 9(a) and 9(b), respectively. Likewise, results for the optimal control profile are depicted in Fig. 9(c). An amplitude 


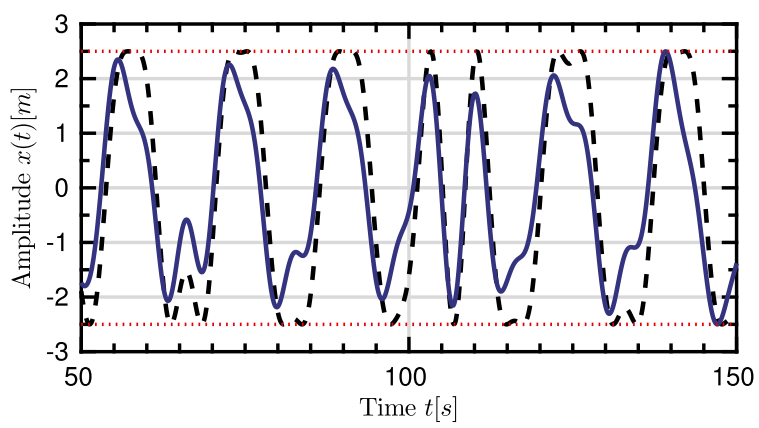

(a)

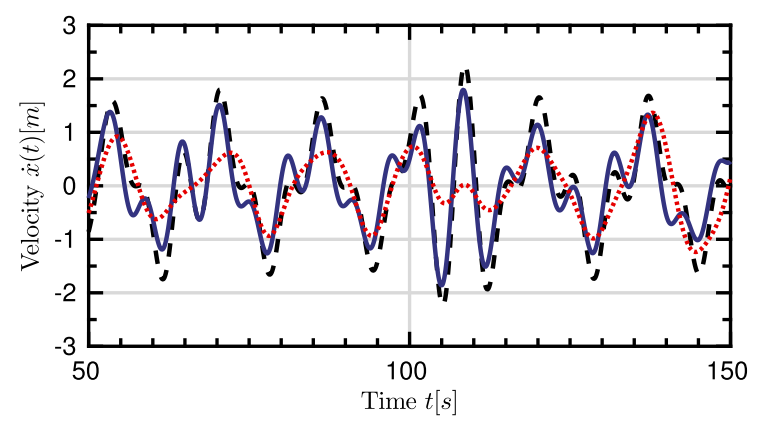

(b)

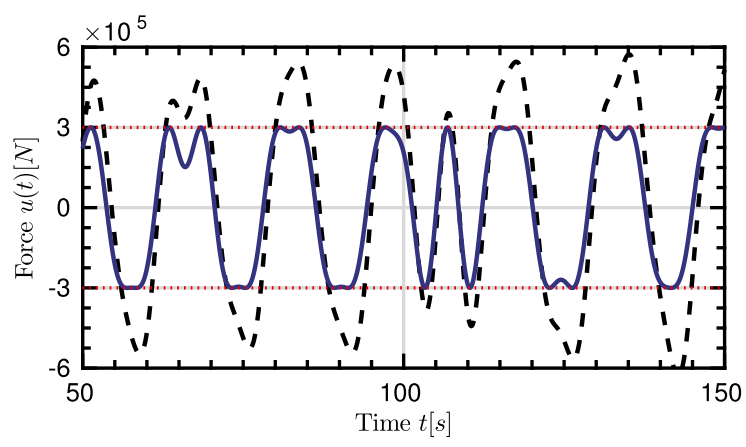

(c)

Fig. 9. Amplitude (a), velocity (b) and optimal PTO force (c) for the irregular waves case when both amplitude and PTO force are constrained (solid-blue) and the case where only the amplitude is constrained (dashed-black). As in the regular waves case, excitation force is presented (scaled, dotted-red) in (b), among with the velocity of the device.

constraint of $X_{\max }=2.5[\mathrm{~m}]$ is considered while, regarding the control input, the constraint is set to a tighter value, namely $U_{\max }=0.3 \times 10^{6}[\mathrm{~N}]$. Simulations are initially performed considering only amplitude limits and then both amplitude and PTO force constraints simultaneously. From Figs. 9(a) and 9(c), it can be immediately appreciated that the constraints are satisfied, according to the control design objective. Moreover, in Fig. 9(c), it can be seen that the "in-phase" optimal energy absorption condition, detailed in Section 5.1, holds for this irregular wave case.

\section{Conclusion}

This study considers the application of a novel mathematical framework, namely the moment-based phasor transform, to compute the steadystate response of a system to develop energy-maximising optimal controllers for WECs. It has been shown that the optimal control formulation, in the moment-domain, is a quadratic program, which depends explicitly only on the control input phasor, allowing the application of well-known computationally efficient algorithms for its solution. It has also been shown that the moment-domain framework can handle the physical constraints of both the device and the PTO system, ensuring the feasibility of the optimal control input computed and securing the durability of the WEC under real sea conditions. Overall, this paper utilises the recent moment-based mathematical framework and uses it to develop a computationally-efficient energy-maximising controller for wave energy converters. Though results are presented for a particular WEC, the approach provides a platform for the effective control of a wide range of WEC devices. Finally, and to further highlight the value of this strategy when comparing to well-known optimal control methods that have been applied to this energy-maximising problem, such as MPC, we note that there exists a close connection between this momentbased optimal control formulation and the MPC-like methods reported in Faedo et al. (2017) (subject of a future paper), which have been shown to outperform MPC in both energy capture and computation time (Genest \& Ringwood, 2016).

\section{Acknowledgments}

This work was supported by the Science Foundation Ireland under Grant No. SFI/13/IA/1886. This work has been partially supported by the European Union's Horizon 2020 Research and Innovation Programme, UK under grant agreement No 739551 (KIOS CoE).

\section{References}

Antoulas, A. C. (2005). Approximation of large-scale dynamical systems. SIAM.

Astolfi, A. (2010). Model reduction by moment matching for linear and nonlinear systems. IEEE Transactions on Automatic Control, 55(10), 2321-2336.

Bacelli, G., \& Ringwood, J. V. (2015). Numerical optimal control of wave energy converters. IEEE Transactions on Sustainable Energy, 6(2), 294-302.

Bacelli, G., Ringwood, J. V., \& Gilloteaux, J.-C. (2011). A control system for a selfreacting point absorber wave energy converter subject to constraints. IFAC Proceedings Volumes, 44(1), 11387-11392.

Boyd, S., \& Vandenberghe, L. (2004). Convex optimization. Cambridge university press.

Brewer, J. (1978). Kronecker products and matrix calculus in system theory. IEEE Transactions on Circuits and Systems, 25(9), 772-781.

Chang, G., Jones, C. A., Roberts, J. D., \& Neary, V. S. (2018). A comprehensive evaluation of factors affecting the levelized cost of wave energy conversion projects. Renewable Energy, 127, 344-354.

Cummins, W. (1962). The impulse response function and ship motions, Tech. rep., DTIC Document.

Davis, A. M. (1998). Linear circuit analysis. PWS Pub..

Edenhofer, O., Pichs-Madruga, R., Sokona, Y., Seyboth, K., Kadner, S., Zwickel, T., et al. (2011). Renewable energy sources and climate change mitigation: Special report of the intergovernmental panel on climate change. Cambridge University Press.

Faedo, N., Olaya, S., \& Ringwood, J. V. (2017). Optimal control, MPC and MPC-like algorithms for wave energy systems: An overview. IFAC Journal of Systems and Control, $1,37-56$.

Fahroo, F., \& Ross, I. M. (2008). Pseudospectral methods for infinite-horizon nonlinear optimal control problems. Journal of Guidance, Control, and Dynamics, 31(4), 927936.

Falcão, A. F. de O. (2010). Wave energy utilization: A review of the technologies. Renewable and Sustainable Energy Reviews, 14(3), 899-918.

Falnes, J. (2002). Ocean waves and oscillating systems: linear interactions including waveenergy extraction. Cambridge university press.

Fusco, F., \& Ringwood, J. V. (2012). A study of the prediction requirements in real-time control of wave energy converters. IEEE Transactions on Sustainable Energy, 3(1), 176184.

Garg, D., Hager, W. W., \& Rao, A. V. (2011). Pseudospectral methods for solving infinitehorizon optimal control problems. Automatica, 47(4), 829-837.

Genest, R., \& Ringwood, J. V. (2016). A critical comparison of model-predictive and pseudospectral control for wave energy devices. Journal of Ocean Engineering and Marine Energy, 2(4), 485-499.

Hasselmann, K. (1973). Measurements of wind wave growth and swell decay during the Joint North Sea Wave Project (JONSWAP). Deutschen Hydrographiques Zeitschrift, 8, 95.

Li, G., \& Belmont, M. R. (2014). Model predictive control of sea wave energy converterspart i: A convex approach for the case of a single device. Renewable Energy, 69, 453463.

Li, G., Weiss, G., Mueller, M., Townley, S., \& Belmont, M. R. (2012). Wave energy converter control by wave prediction and dynamic programming. Renewable Energy, 48, 392403. 
Meier, H., \& Nowacki, H. (1987). Interpolating curves with gradual changes in curvature. Computer Aided Geometric Design, 4(4), 297-305.

Mérigaud, A., \& Ringwood, J. V. (2017). Free-surface time-series generation for wave energy applications. IEEE Journal of Oceanic Engineering.

Mork, G., Barstow, S., Kabuth, A., \& Pontes, M. T. (2010). Assessing the global wave energy potential. In ASME 2010 29th international conference on ocean, offshore and arctic engineering (pp. 447-454). American Society of Mechanical Engineers.

Nielsen, K. M., Pedersen, T. S., Andersen, P., \& Ambühl, S. (2017). Optimizing control of wave energy converter with losses and fatigue in power take off. IFAC-PapersOnLine, 50(1), 14680-14685, 20th IFAC World Congress.

Nilsson, J. W., \& Riedel, S. A. (2015). Electric circuits. Pearson.

Ogilvie, T. F. (1964). Recent progress toward the understanding and prediction of ship motions. In 5th symposium on naval hydrodynamics, Vol. 1 (pp. 2-5). Norway: Bergen.

Padoan, A., Scarciotti, G., \& Astolfi, A. (2017). A geometric characterization of the persistence of excitation condition for the solutions of autonomous systems. IEEE Transactions on Automatic Control, 62(11), 5666-5677.

Pelc, R., \& Fujita, R. M. (2002). Renewable energy from the ocean. Marine Policy, 26(6), 471-479.

Reinsch, C. H. (1967). Smoothing by spline functions. Numerische Mathematik, 10(3), 177183.
Richter, M., Magaña, M. E., Sawodny, O., \& Brekken, T. K. (2014). Power optimisation of a point absorber wave energy converter by means of linear model predictive control. IET Renewable Power Generation, 8(2), 203-215.

Ringwood, J. V., Bacelli, G., \& Fusco, F. (2014). Energy-maximizing control of waveenergy converters: The development of control system technology to optimize their operation. IEEE Control Systems, 34(5), 30-55.

Scarciotti, G., \& Astolfi, A. (2015). Characterization of the moments of a linear system driven by explicit signal generators. In American control conference (ACC), 2015 (pp. 589-594). IEEE.

Scarciotti, G., \& Astolfi, A. (2016a). Model reduction by matching the steady-state response of explicit signal generators. IEEE Transactions on Automatic Control, 61(7), 19952000.

Scarciotti, G., \& Astolfi, A. (2016b). Moment-based discontinuous phasor transform and its application to the steady-state analysis of inverters and wireless power transfer systems. IEEE Transactions on Power Electronics, 31(12), 8448-8460.

Scarciotti, G., \& Astolfi, A. (2017). Nonlinear model reduction by moment matching. Foundations and Trends in Systems and Control, 4(3-4), 224-409.

Van Loan, C. F. (2000). The ubiquitous Kronecker product. Journal of Computational and Applied Mathematics, 123(1), 85-100.

Zhang, F. (2011). Matrix theory: basic results and techniques. Springer Science \& Business Media. 